\title{
On the shadow of squashed families of $k$-sets
}

\author{
Frédéric Maire \\ maire@fit.qut.edu.au \\ Neurocomputing Research Center \\ Queensland University of Technology \\ Box 2434 Brisbane Qld 4001, Australia
}

\begin{abstract}
The shadow of a collection $\mathcal{A}$ of $k$-sets is defined as the collection of the $(k-1)$-sets which are contained in at least one $k$-set of $\mathcal{A}$. Given $|\mathcal{A}|$, the size of the shadow is minimum when $\mathcal{A}$ is the family of the first $k$-sets in squashed order (by definition, a $k$-set $A$ is smaller than a $k$-set $B$ in the squashed order if the largest element of the symmetric difference of $A$ and $B$ is in $B$ ). We give a tight upper bound and an asymptotic formula for the size of the shadow of squashed families of $k$-sets.
\end{abstract}

Submitted: January 15, 1995; Accepted: August 25, 1995.

AMS Subject Classification. 04A20,03E05,05A20.

\section{Introduction}

A hypergraph is a collection of subsets (called edges) of a finite set $S$. If a hypergraph $\mathcal{A}$ is such that $A_{i}, A_{j} \in \mathcal{A}$ implies $A_{i} \nsubseteq A_{j}$, then $\mathcal{A}$ is called an antichain. In other words $\mathcal{A}$ is a collection of incomparable sets. Antichains are also known under the names simple hypergraph or clutter.

The shadow of a collection $\mathcal{A}$ of $k$-sets (set of size $k$ ) is defined as the collection of the $(k-1)$-sets which are contained in at least one $k$-set of $\mathcal{A}$. The shadow of $\mathcal{A}$ is denoted by $\Delta(\mathcal{A})$.

In the following we assume that $S$ is a set of integers. The squashed order is defined on the the set of $k$-sets. Given two $k$-sets $A$ and $B$, we say that $A$ is smaller than $B$ in the squashed order if the largest element of the symmetric difference of $A$ and $B$ is in $B$. The first 3 -sets in the squashed order are

$$
\{1,2,3\},\{1,2,4\},\{1,3,4\},\{2,3,4\},\{1,2,5\},\{1,3,5\}, \cdots
$$

Let $F_{k}(x)$ denote the family of the first $x k$-sets in the squashed order. We will prove the following. 
Theorem 1 If $x \leq\left(\begin{array}{l}n \\ k\end{array}\right)$ then

$$
\left|\Delta\left(F_{k}(x)\right)\right| \leq k x-x(x-1) \times q_{n, k} \text { where } q_{n, k}=\frac{k}{\left(\begin{array}{l}
n \\
k
\end{array}\right)-1} \times \frac{n-k}{n-k+1}
$$

Equality holds when $x=0$ or $x=\left(\begin{array}{l}n \\ k\end{array}\right)$.

Theorem 2 When $x \rightarrow \infty,\left|\Delta\left(F_{k}(x)\right)\right| \sim \frac{k}{\sqrt[k]{k !}} x^{1-\frac{1}{k}}$

The squashed order is very useful when dealing with the size of the shadow of a collection of $k$-sets. The main result is that if you want to minimize the shadow then you have to take the first sets in the squashed order. This is a consequence of the Kruskal-Katona theorem [4, 3]. Before stating their theorem, recall the definition of the $l$-binomial representation of a number.

Theorem 3 Given positive integers $x$ and $l$, there exists a unique representation of $x$ (called the l-binomial representation) in the form

$$
x=\left(\begin{array}{c}
x_{l} \\
l
\end{array}\right)+\left(\begin{array}{c}
x_{l-1} \\
l-1
\end{array}\right)+\cdots+\left(\begin{array}{c}
x_{t} \\
t
\end{array}\right)
$$

where $x_{l}>x_{l-1}>\cdots>x_{t} \geq t$.

See [1] or [2] for more details.

Theorem 4 (Kruskal-Katona) Let $\mathcal{A}$ be a collection of l-sets, and suppose that the l-binomial representation of $|\mathcal{A}|$ is

$$
|\mathcal{A}|=\left(\begin{array}{c}
x_{l} \\
l
\end{array}\right)+\left(\begin{array}{c}
x_{l-1} \\
l-1
\end{array}\right)+\cdots+\left(\begin{array}{c}
x_{t} \\
t
\end{array}\right)
$$

where $x_{l}>x_{l-1}>\cdots>x_{t} \geq t$. Then

$$
|\Delta(\mathcal{A})| \geq\left(\begin{array}{c}
x_{l} \\
l-1
\end{array}\right)+\left(\begin{array}{c}
x_{l-1} \\
l-2
\end{array}\right)+\cdots+\left(\begin{array}{c}
x_{t} \\
t-1
\end{array}\right)
$$

There is equality when $\mathcal{A}$ is the collection of the first $|\mathcal{A}|$ l-sets in the squashed order.

Though the above theorem gives the exact values of the shadow when the antichain is squashed, it is awkward to manipulate. Because of this, theorem 1 may be more useful for some problems such as those of construction of completely separating systems (see [5], for example). 


\section{Proofs}

\subsection{Proof of theorem 1}

We need a few lemmas before proving theorem 1 .

Lemma 1 The inequality of theorem 1 holds when $n \leq 6$.

Proof of lemma 1: Done by computer check. Can be done by hand too.

Lemma 2 The inequality of theorem 1 holds when $k=1$.

Proof of lemma 2: We have $q_{n, 1}=1 / n$. So the inequality to prove is;

$$
\left|\Delta\left(F_{1}(x)\right)\right| \leq x-x(x-1) \times \frac{1}{n}
$$

The right hand side of the inequality can be rewritten as

$$
\frac{x}{n}(n-x+1)
$$

As $\left|\Delta\left(F_{1}(x)\right)\right|$ is equal to 1 (because $\Delta\left(F_{1}(x)\right)=\{\emptyset\}$ ), all we have to prove is that

$$
\frac{n}{x} \leq n-x+1
$$

i.e.

$$
x^{2}-(n+1) x+n \leq 0
$$

The zeroes of this polynomial are 1 and $n$. This implies that for $x$ in the interval $\left[1,\left(\begin{array}{l}n \\ 1\end{array}\right)\right]$, the inequality holds.

Lemma 3 The inequality of theorem 1 holds when $k=n-1$.

Proof of lemma 3: We have $q_{n, n-1}=\frac{1}{2}$. So the inequality to prove is;

$$
\left|\Delta\left(F_{n-1}(x)\right)\right| \leq x\left[n-1-\frac{x-1}{2}\right]
$$

The value of $x$ is in the range $[1, n]$. If $x=n$ then both sides of the inequality are equal to $\left(\begin{array}{l}n \\ 2\end{array}\right)$. Now, assume that $x$ is in the range $[1, n-1]$. The $(n-1)$-binomial representation of $x$ is:

$$
x=\left(\begin{array}{c}
x_{n-1} \\
n-1
\end{array}\right)+\left(\begin{array}{c}
x_{n-2} \\
n-2
\end{array}\right)+\cdots+\left(\begin{array}{c}
x_{t} \\
t
\end{array}\right)
$$


where $x_{n-1}>x_{n-2}>\cdots>x_{t} \geq t$. As $x \leq n-1$, we have $x_{n-1}=n-1$. And, therefore $x_{n-i}=n-i$ for all $i \in[1, n-t]$. Hence $x=n-t$. Because of the $(n-1)$-binomial representation of $x$, the size of the shadow of $F_{n-1}(x)$ is given by the formula:

$$
\left|\Delta\left(F_{n-1}(x)\right)\right|=\left(\begin{array}{l}
n-1 \\
n-2
\end{array}\right)+\left(\begin{array}{l}
n-2 \\
n-3
\end{array}\right)+\cdots+\left(\begin{array}{c}
t \\
t-1
\end{array}\right)
$$

i.e.

$$
\left|\Delta\left(F_{n-1}(x)\right)\right|=\left(\begin{array}{c}
n-1 \\
1
\end{array}\right)+\left(\begin{array}{c}
n-2 \\
1
\end{array}\right)+\cdots+\left(\begin{array}{l}
t \\
1
\end{array}\right)
$$

Finally, we have

$$
\left|\Delta\left(F_{n-1}(x)\right)\right|=\frac{n(n-1)}{2}-\frac{t(t-1)}{2}=\frac{1}{2}(n-t)(n+t-1)
$$

As $x=n-t$. By substituting $n-x$ to $t$ in the right hand side, we find that

$$
\left|\Delta\left(F_{n-1}(x)\right)\right|=x\left[n-1-\frac{x-1}{2}\right]
$$

Which is what we wanted to prove.

Lemma 4 The inequality of theorem 1 holds when $k=n$.

Proof of lemma 4: obvious.

Lemma 5 The function $n \longmapsto q_{n, k}$ is decreasing on $[k+1, \infty]$.

\section{Proof of lemma 5:}

$$
q_{n+1, k}-q_{n, k}=\frac{k}{\left(\begin{array}{c}
n+1 \\
k
\end{array}\right)-1} \times \frac{n+1-k}{n+2-k}-\frac{k}{\left(\begin{array}{l}
n \\
k
\end{array}\right)-1} \times \frac{n-k}{n+1-k}
$$

which has the same sign as

$$
k(n+1-k)^{2} \times\left(\left(\begin{array}{l}
n \\
k
\end{array}\right)-1\right)-k(n-k)(n+2-k) \times\left(\left(\begin{array}{c}
n+1 \\
k
\end{array}\right)-1\right)
$$

which has the same sign as

$$
(n+1-k)^{2} \times\left(\left(\begin{array}{l}
n \\
k
\end{array}\right)-1\right)-(n-k)(n+2-k) \times\left(\left(\begin{array}{l}
n \\
k
\end{array}\right)+\left(\begin{array}{c}
n \\
k-1
\end{array}\right)-1\right)
$$


THE ELECTRONIC JOURNAL OF COMBINATORICS 2 (1995) \#R16

$$
\begin{aligned}
& =\left(\begin{array}{l}
n \\
k
\end{array}\right)-1-(n-k)(n-k+2) \times\left(\begin{array}{c}
n \\
k-1
\end{array}\right) \\
& =\left(\begin{array}{l}
n \\
k
\end{array}\right)-1-\left(\begin{array}{l}
n \\
k
\end{array}\right) \frac{k(n-k)(n-k+2)}{n-k+1}<0
\end{aligned}
$$

To prove theorem 1, we use a double induction on $k$ then $n$. The case $k=1$ has been considered in lemma 2. If $x \leq\left(\begin{array}{c}n-1 \\ k\end{array}\right)$ then as the function $n \longmapsto q_{n, k}$ is decreasing, using the induction hypothesis we are done. Thus, we can assume that $x=\left(\begin{array}{c}n-1 \\ k\end{array}\right)+j$ with $j \leq\left(\begin{array}{c}n-1 \\ k-1\end{array}\right)$. It is a classical result (see [2] or [1]) that

$$
\left|\Delta\left(F_{k}(x)\right)\right|=\left(\begin{array}{c}
n-1 \\
k-1
\end{array}\right)+\left|\Delta\left(F_{k-1}(j)\right)\right|
$$

By induction hypothesis

$$
\left|\Delta\left(F_{k-1}(j)\right)\right| \leq j(k-1)-j(j-1) \times q_{n-1, k-1}
$$

Combining these inequalities we get:

\section{Claim 1}

$$
\left|\Delta\left(F_{k}(x)\right)\right| \leq\left(\begin{array}{l}
n-1 \\
k-1
\end{array}\right)+j(k-1)-j(j-1) q_{n-1, k-1}
$$

If theorem 1 is true then $\left|\Delta\left(F_{k}(x)\right)\right| \leq k x-x(x-1) \times q_{n, k}$ with equality when $j=\left(\begin{array}{l}n-1 \\ k-1\end{array}\right)$. Hence, to prove theorem 1 it is sufficient to prove that we have:

$$
\left(\begin{array}{l}
n-1 \\
k-1
\end{array}\right)+j(k-1)-j(j-1) q_{n-1, k-1} \leq k x-x(x-1) \times q_{n, k}
$$

As $k\left(\begin{array}{c}n-1 \\ k\end{array}\right)=(n-k)\left(\begin{array}{c}n-1 \\ k-1\end{array}\right)$ and $x=\left(\begin{array}{c}n-1 \\ k\end{array}\right)+j,(\star)$ is equivalent to

$$
x(x-1) q_{n, k} \leq(n-k-1)\left(\begin{array}{l}
n-1 \\
k-1
\end{array}\right)+j+j(j-1) q_{n-1, k-1}
$$

To simplify the expressions we introduce some new variables. Let $q_{0}=q_{n, k}$ and $q_{1}=q_{n-1, k-1}$. Let $y=\left(\begin{array}{l}n-1 \\ k-1\end{array}\right)$. We will use later the facts that $\left(\begin{array}{l}n \\ k\end{array}\right)=\frac{n}{k} y$, and that $\left(\begin{array}{c}n-1 \\ k\end{array}\right)=\frac{n-k}{k} y$. With this notation $(\star)$ is equivalent to

$$
x(x-1) q_{0} \leq(n-k-1) y+j(j-1) q_{1}+j
$$


As $x=\frac{n-k}{k} y+j$, we have

$$
x(x-1) q_{0}=q_{0} j^{2}+q_{0}\left(2 \frac{n-k}{k} y-1\right) j+q_{0}\left(\frac{n-k}{k} y\right)^{2}-\frac{n-k}{k} y q_{0}
$$

Therefore, $(\star)$ is equivalent to

$0 \leq j^{2}\left(q_{1}-q_{0}\right)-j\left(-1+q_{1}-q_{0}+2 \frac{n-k}{k} y q_{0}\right)+(n-k-1) y-q_{0}\left(\frac{n-k}{k} y\right)^{2}+\frac{n-k}{k} y q_{0}$

Finally we have,

Claim $2(\star)$ is equivalent to

$0 \leq j^{2}\left(q_{1}-q_{0}\right)-j\left(-1+q_{1}-q_{0}+2 \frac{n-k}{k} y q_{0}\right)+(n-k-1) y+q_{0} \frac{n-k}{k} y\left(1-\frac{n-k}{k} y\right)$

Let $\Phi(j)=j^{2}\left(q_{1}-q_{0}\right)-j\left(-1+q_{1}-q_{0}+2 \frac{n-k}{k} y q_{0}\right)+(n-k-1) y+q_{0} \frac{n-k}{k} y\left(1-\frac{n-k}{k} y\right)$. We will prove that this polynomial in $j$ is positive on the interval $\left[0,\left(\begin{array}{l}n-1 \\ k-1\end{array}\right)\right]$, by proving that $\Phi^{\prime \prime} \geq 0, \Phi^{\prime}(y) \leq 0$ and $\Phi(y)=0$. Let's prove that $\Phi^{\prime \prime}=q_{1}-q_{0}$ is positive.

$$
q_{0}-q_{1}=\left[\frac{k}{\left(\begin{array}{l}
n \\
k
\end{array}\right)-1}-\frac{k-1}{\left(\begin{array}{l}
n-1 \\
k-1
\end{array}\right)-1}\right] \frac{n-k}{n-k+1}
$$

i.e.

$$
q_{0}-q_{1}=\left[\frac{k}{\frac{n}{k} y-1}-\frac{k-1}{y-1}\right] \frac{n-k}{n-k+1}
$$

The sign of $q_{0}-q_{1}$ is the same as the sign of

$$
k(y-1)-(k-1)\left(\frac{n}{k} y-1\right)=k y-k-n y+k+\frac{n}{k} y-1=y\left(k-n+\frac{n}{k}\right)-1
$$

Notice that $k-n+\frac{n}{k}$ is negative because $k \in[2, n-2]$. Indeed, the sign of $k-n+\frac{n}{k}$ is the same as the sign of $k^{2}-n k+n$. It's easy to check that this polynomial in $k$ is negative on $[2, n-1]$ as soon as $n \geq 5$. Hence, $q_{0}-q_{1}$ is negative.

Let's check that $(\star)$ becomes an equality when $j$ takes the value of $y=\left(\begin{array}{l}n-1 \\ k-1\end{array}\right)$. By substituting $\left(\begin{array}{l}n \\ k\end{array}\right)$ to $x$ in the right hand side of the inequality of theorem 1 , we get $\left(\begin{array}{c}n \\ k-1\end{array}\right)$ as expected. By substituting $y=\left(\begin{array}{l}n-1 \\ k-1\end{array}\right)$ to $j$ in the inequality of claim 1 , we obtain also $\left(\begin{array}{c}n \\ k-1\end{array}\right)$ (use the induction hypothesis that $\left|\Delta\left(F_{k-1}(y)\right)\right|=\left(\begin{array}{l}n-1 \\ k-2\end{array}\right)$ ). This implies that $\left(\begin{array}{l}n-1 \\ k-1\end{array}\right)$ is a root of the polynomial $\Phi(j)$.

To finish the proof of theorem 1 we will prove that $y=\left(\begin{array}{l}n-1 \\ k-1\end{array}\right)$ is the smaller root of $\Phi(j)$, by showing that at that point the derivative of $\Phi(j)$ is negative. This will sufficient as we already know that the second derivative is positive. We have

$$
\Phi^{\prime}(y)=2 y\left(q_{1}-q_{0}\right)-\left(-1+q_{1}-q_{0}+2 \frac{n-k}{k} y q_{0}\right)
$$


$\Phi^{\prime}(y) \leq 0$ is equivalent to

$$
2 y\left(q_{1}-q_{0}\right) \leq-1+q_{1}-q_{0}+2 \frac{n-k}{k} y q_{0}
$$

which is equivalent to

$$
2 y\left(\frac{k-1}{y-1}-\frac{k}{\frac{n}{k} y-1}\right) \frac{n-k}{n-k+1} \leq-1+q_{1}-q_{0}+2 \frac{n-k}{k} y \frac{k}{\frac{n}{k} y-1} \frac{n-k}{n-k+1}
$$

which is equivalent to

$$
2 y\left(\frac{k-1}{y-1}-\frac{k^{2}}{n y-k}\right)+\frac{n-k+1}{n-k} \leq\left(q_{1}-q_{0}\right) \frac{n-k+1}{n-k}+\frac{2(n-k) k y}{n y-k}
$$

i.e.

$$
\frac{2 y(k-1)}{y-1}+\frac{n-k+1}{n-k} \leq\left(q_{1}-q_{0}\right) \frac{n-k+1}{n-k}+\frac{2 n k y}{n y-k}
$$

It is sufficient to prove that

$$
\frac{2 y(k-1)}{y-1}+\frac{3}{2} \leq \frac{2 n k y}{n y-k}
$$

The left hand side is equal to $2 k-\frac{1}{2}+\frac{2(k-1)}{y-1}$. The right hand side is equal to $2 k+\frac{2 k^{2}}{n y-k}$. The function $t \mapsto \frac{-1}{2}+\frac{2(k-1)}{t-1}$ is negative as soon as $t \geq 4(k-1)+1$. As $n \geq 7$ and $k \in[2, n-2]$, we have $y=\left(\begin{array}{l}n-1 \\ k-1\end{array}\right) \geq 4(k-1)+1$. Therefore,

$$
\frac{2 y(k-1)}{y-1}+3 / 2 \leq \frac{2 n k y}{n y-k}
$$

This finishes the proof of theorem 1.

\subsection{Proof of theorem 2}

Consider the $k$-binomial representation of $x$ :

$$
x=\left(\begin{array}{c}
x_{k} \\
k
\end{array}\right)+\left(\begin{array}{c}
x_{k-1} \\
k-1
\end{array}\right)+\cdots+\left(\begin{array}{c}
x_{t} \\
t
\end{array}\right) \text { where } x_{k}>x_{k-1}>\cdots>x_{t} \geq t
$$

It is easy to prove that

$$
\text { when } x \rightarrow \infty, \quad x \sim\left(\begin{array}{c}
x_{k} \\
k
\end{array}\right) \text { and similarly }, \quad\left|\Delta\left(F_{k}(x)\right)\right| \sim\left(\begin{array}{c}
x_{k} \\
k-1
\end{array}\right)
$$


As $x \sim\left(\begin{array}{c}x_{k} \\ k\end{array}\right)$, we have $x \sim \frac{x_{k}{ }^{k}}{k !}$. This implies that $x_{k} \sim(x(k !))^{\frac{1}{k}}$. Therefore

$$
\frac{\left|\Delta\left(F_{k}(x)\right)\right|}{x} \sim \frac{\left(\begin{array}{c}
x_{k} \\
k-1
\end{array}\right)}{\left(\begin{array}{c}
x_{k} \\
k
\end{array}\right)} \sim \frac{k}{x_{k}-k+1}
$$

Hence $\quad \frac{\left|\Delta\left(F_{k}(x)\right)\right|}{x} \sim \frac{k}{(x(k !))^{\frac{1}{k}}} \square$

\section{References}

[1] Anderson I. : Combinatorics of finite sets, Oxford science publication, 1987.

[2] Berge C. : Graphs and Hypergraphs, North-Holland, 1985.

[3] Katona, G. O. H. (1966) : A theorem on finite sets. In 'Theory of Graphs'. Proc. Colloq. Tihany, 1966, pp. 187-207. Akademia Kiado. Academic Press, New York.

[4] Kruskal, J. B. (1963) : The number of simplices in a complex. In 'Mathematical optimization techniques' (ed. R. Bellman), pp. 251-78. University of California Press, Berkeley.

[5] Ramsey, C., Roberts I. (1994) : Minimal completely separating systems of $k$-sets. To appear in 'Proc. Colloq. of the 20th Australasian Conference on Combinatorial Mathematics', Auckland 1994. 